\title{
A Two-Class Stochastic Network Equilibrium Model under Adverse Weather Conditions
}

\author{
Chenming Jiang $\mathbb{D}^{1},{ }^{1}$ Linjun Lu $\mathbb{D}^{2}{ }^{2}$ Junliang He, ${ }^{1,3}$ and Caimao Tan ${ }^{1}$ \\ ${ }^{1}$ China Institute of FTZ Supply Chain, Shanghai Maritime University, Shanghai 201306, China \\ ${ }^{2}$ School of Naval Architecture, Ocean and Civil Engineering, Shanghai Jiao Tong University, Shanghai 200240, China \\ ${ }^{3}$ Engineering Research Center of Container Supply Chain Technology, Ministry of Education, Shanghai Maritime University, \\ Shanghai 201306, China \\ Correspondence should be addressed to Linjun Lu; linjunlu@sjtu.edu.cn
}

Received 22 December 2018; Revised 14 July 2019; Accepted 13 September 2019; Published 22 June 2020

Academic Editor: Monica Menendez

Copyright (C) 2020 Chenming Jiang et al. This is an open access article distributed under the Creative Commons Attribution License, which permits unrestricted use, distribution, and reproduction in any medium, provided the original work is properly cited.

\begin{abstract}
Adverse weather condition is one of the inducements that lead to supply uncertainty of an urban transportation system, while travelers' multiple route choice criteria are the nonignorable reason resulting in demand uncertainty. This paper proposes a novel stochastic traffic network equilibrium model considering impacts of adverse weather conditions on roadway capacity and route choice criteria of two-class mixed roadway travellers on demand modes, in which the two-class route choice criteria root in travelers' different network information levels (NILs). The actual route travel time (ARTT) and perceived route travel time (PRTT) are considered as the route choice criteria of travelers with perfect information (TPI) and travelers with bounded information (TBI) under adverse weather conditions, respectively. We then formulate the user equilibrium (UE) traffic assignment model in a variational inequality problem and propose a solution algorithm. Numerical examples including a small triangle network and the Sioux Falls network are presented to testify the validity of the model and to clarify the inner mechanism of the two-class UE model under adverse weather conditions. Managerial implications and applications are also proposed based on our findings to improve the operation efficiency of urban roadway network under adverse weather conditions.
\end{abstract}

\section{Introduction}

Uncertainties of transportation networks are logically derived from supply side (roadway capacity fluctuation) and demand side (travel demand variation) [1-4], which can also be considered as exogenous and endogenous sources [5], respectively. On account of the overwhelming majority of transportation modes being directly exposed in the atmospheric environment, the total process of traffic activity is inevitably more or less subject to adverse weather conditions, such as rainfall, snow, fog, and high winds, etc. [6]. Therefore, adverse weather conditions are widely recognized as a primary inducement markedly generating the uncertainties of roadway capacity. On the other side, travel demand uncertainties mainly come from the temporal factors, such as time of day [7], day of week or season effects. Note that travelers' heterogeneous route choice criteria are also considered as a nonignorable reason $[8,9]$ resulting in demand uncertainty. Multiclass route choice behavior throughout population usually root from travelers' differentiated levels of attainable network information about the present network scenarios. Finally, the interaction existing between traffic supply and demand $[10,11]$ of the unreliable or uncertain transport system can change traveler's behavior and then arouse the spatial and temporal fluctuation of traffic demand.

As a vital inducement to the travel time uncertainty, adverse weather condition has been understanding and modeling its relationship with travelers' route choice decisions [12]. Therefore, in this study, we will focus on the impact of adverse weather conditions on network equilibrium traffic assignment model considering traffic network uncertainty. Lam et al. [13] and Shao et al. [14] are believed to be the first studies who implicitly incorporated the impacts of adverse weather (i.e., rainfall) on both free flow speed and roadway capacity into the Bureau of Public Roads (BPR) function, which was followed and transplanted to multi-modal transport network problems 
under adverse weather conditions by Sumalee et al. [15] and Uchida et al. [8]. However, few works have been conducted in the subdivision of travelers' various route choice criteria including the impacts of different levels of information penetration under weather conditions. In the SUE model considering the effects of rainfall intensity on the road performance proposed by Lam et al. [13], they assume that travelers are homogeneous that all travelers are considered as undifferentiated ones who choose their routes with the minimum perceived effective travel time based on the occurring probability of weather scenarios (i.e., no rain/light rain, normal rain, amber rainstorm, etc.). Nowadays, however, with the aid of Advanced Traveler Information System (ATIS), some motorists can obtain the realtime traffic information of the current network, and then make their optimal routing choices based on the recommended actual shortest routes. Therefore, in this study, to model the network equilibrium problem more realistically, we intuitively assume that the traveler population are divided into two exclusive classes by their different network information levels (NILs) under adverse weather conditions: (1) with the equipment with ATIS, one part of travelers who can acquire a perfect knowledge of actual, scenario-specific network condition (i.e., travelers with perfect information, TPI) would fully choose the recommended actual shortest route with minimum actual travel cost, which accords with the wisdom of conventional user equilibrium [16], (2) analogous to the traveler feature that depicted in Lam et al. [13], the other part of travelers, who are clustered without the actual information of the network and named as travelers with bounded information (TBI), choose their routes with minimum perceived travel cost according to their individual travelling experiences and perceptions on the information of weather forecast. In Lam et al. [13] and Shao et al. [14], all the travelers considered were those who can acquire the weather forecast information, while those who are armed with ATIS and can acquire the actual link travel time in the network were not considered. This paper contributes to the literature by extending the works of Lam et al. [13] and Shao et al. [14] by simultaneously considering both of the two user classes, i.e., TBI (in [13, 14] and TPI with ATIS (in the traditional Wardrop UE model). Besides, to focus on a traffic planning level, this study is to propose a static UE model without considering travelers' attitudes towards risk (i.e., travel time reliability), rather than a SUE one.

The remainder of this paper is organized as follows. Section 2 develops a brief problem description of this study. Section 3 elaborately depicts the UE model in a mathematical formulation. Corresponding solution algorithm is proposed in Section 4. In Section 5, two numerical examples are presented to illustrate the validity of the proposed model and solution algorithm. Finally, the conclusions of the study and recommendations for future perspective are given in Section 6.

\section{Problem Descriptions}

Define a stochastic traffic system $\Pi=(\boldsymbol{N}, \boldsymbol{U})$ consisting of a traffic network subsystem $\boldsymbol{N}=(\boldsymbol{V}, \boldsymbol{A}, \boldsymbol{S}, \boldsymbol{P})$ and a two-class traveler subsystem $\boldsymbol{U}=(\boldsymbol{J})$. For stochastic traffic networks $\boldsymbol{N}, \boldsymbol{V}$ is the node set, $\boldsymbol{A}$ is the link set, $\boldsymbol{S}$ is the network scenario set and $\boldsymbol{P}$ is the occurring probability set related to network scenarios. For users $\boldsymbol{U}, \boldsymbol{J}$ is the set of travelers' network information levels (NILs).

In meteorology, adverse weather conditions refer to various categories of local weather events that are destructive or not conducive to human production and activities, which differentiate with each other in disaster-causing mechanism, such as rainfall, snow, fog, high winds, and extreme temperatures etc., While in this transportation-related study, adverse weather conditions are further defined as those local weather events that can directly degrade roadway capacity and may affect travelers' route choice behavior. Meanwhile, for a specific adverse weather condition, we define the network scenariosas subdivisions by different intensity levels under a certain adverse weather condition. For example, for a certain adverse weather condition, say, rainfall, there may be five network scenarios in China consisting of light rain, moderate rain, heavy rain, rainstorm, and heavy rainstorm that are classified by different rainfall intensity levels (mm per hour). To model the traffic network equilibrium problem, the foundation is to elaborate the travelers' route choice behavior (or criterion) under different network scenarios. In the traditional user equilibrium [16], travelers are assumed to know the network information perfectly and choose their routes according to actual minimum disutilities. Nowadays, by being equipped with ATIS (e.g., some prevailing cellphone navigation apps), travelers with heterogeneous traveling experience are elevated to ones of homogeneous, nearly-perfect insight on actual network situation. Without the help of ATIS, however, travelers are generally unrealistic to grasp perfect knowledge of all route travel costs in the network. Therefore, in this paper, the notion of two-class users consisting of travelers with perfect information (TPI) and bounded information (TBI) is incorporated to our model to describe roadway users' heterogeneity in NILs. All travelers (TPI and TBI) choose their own travel routes in terms of their individual perceived route travel time (PRTT). To be specific, the PRTT of TPI is the actual route travel time (ARTT) acquired from the ATIS, while the PRTT of TBI is derived from travelers' perception on the accuracy of weather forecast information and the ARTT under each network scenario.

The majority of notations used throughout the paper are listed in Table 1 unless otherwise specified.

\section{Model and Formulation}

3.1. Link Cost Function under Adverse Weather Conditions. The impacts of adverse weather conditions on the road performance, which can intuitively be learned as the deterioration of the visibility and pavement friction, would degrade the roadway performance by decreasing the free-flow speed and ideal link capacity. To capture these impacts, a generalized Bureau of Public Roads (GBPR) function [14] is proposed based on the Bureau of Public Roads (BPR) function as follows:

$$
\begin{aligned}
t_{a}^{s}\left(i_{s}, x_{a}\right) & =t_{a}^{s, 0}\left(i_{s}\right)\left(1+\alpha\left(\frac{x_{a}}{c_{a}^{s}\left(i_{s}\right)}\right)^{\beta}\right) \\
& =g_{t}^{a}\left(i_{s}\right) t_{a}^{0}\left(1+\alpha\left(\frac{x_{a}}{g_{c}^{a}\left(i_{s}\right) c_{a}^{0}}\right)^{\beta}\right) \quad \forall a \in A, \quad s \in S
\end{aligned}
$$


TABLE 1: The notion list.

\begin{tabular}{|c|c|}
\hline Sets & \\
\hline$V$ & Set of nodes, $V=\{\mathrm{v}\}$ \\
\hline$A$ & Set of links, $A=\{a\}$ \\
\hline$S$ & Set of network scenarios, $S=\{s\}$ \\
\hline$P$ & $\begin{array}{l}\text { Set of occurring probabilities of network } \\
\text { scenarios, } P=\{p\}\end{array}$ \\
\hline$J$ & $\begin{array}{l}\text { Set of travelers' network information levels, } \\
\quad J=\{j\} \text {; for TPI, } j=1 \text {; for TBI, } j=2\end{array}$ \\
\hline$W$ & Set of O-D (Origin-Destination) pairs, $\mathrm{W}=\{w\}$ \\
\hline$R$ & Set of routes, $R=\{r\}$ \\
\hline$R_{w}$ & Set of routes connecting O-D pairs $w, R_{w} \subset R$ \\
\hline$D$ & $\begin{array}{l}\text { Scenario-specific conditional probability set of } \\
\text { TBI, } D=\left\{d_{\bar{p}_{1} / 1}, d_{\vec{p}_{2} / 2}, \ldots, d_{\bar{p}_{s} / s}, \ldots, d_{\bar{p}_{|S|} /|S|}\right\}\end{array}$ \\
\hline Para & \\
\hline$i_{s}$ & $\begin{array}{l}\text { Expected rainfall intensity (e.g., } \mathrm{mm} / \mathrm{h} \text { ) in } \\
\text { scenario } s \text {. }\end{array}$ \\
\hline$c_{a}^{0}$ & Ideal or maximum capacity of link $a$ \\
\hline$c_{a}^{s}$ & Capacity of link $a$ under scenario $s$ \\
\hline$q_{w}$ & Mean O-D demand between O-D pair $w$ \\
\hline$x_{a}$ & Travel flow on link $a$ \\
\hline$t_{a}^{s}$ & Actual travel cost on link $a$ under scenario $s$ \\
\hline$r$ & Route index, $\forall r \in R$ \\
\hline$t_{r w}^{s}$ & $\begin{array}{l}\text { Travel time on route } r \text { between O-D pair } w \text { under } \\
\text { scenario } s\end{array}$ \\
\hline$f_{r w}$ & Travel flow on route $r$ between O-D pair $w$ \\
\hline$t_{1 r w}^{s}$ & $\begin{array}{c}\text { Actual route travel time (ARTT) on route } r \\
\text { between O-D pair } w \text { in scenario } s\end{array}$ \\
\hline$\delta_{\text {arw }}$ & $\begin{array}{l}\text { Link-route incidence parameter; } \delta_{\text {arw }}=1 \text {, } \\
\text { if link } a \text { is on route } r \text { that connecting } \mathrm{O}-\mathrm{D} \text { pair } \\
w \text {, zero otherwise }\end{array}$ \\
\hline
\end{tabular}

in which $\alpha=0.15, \beta=4.0$, and $i_{s}$ is the adverse weather intensity under adverse weather scenario (AWS) $s$, e.g., the hourly average rainfall intensity (i.e., $\mathrm{mm} / \mathrm{h}$ ) of moderate rain.

$$
\begin{aligned}
& t_{a}^{s, 0}\left(i_{s}\right)=g_{t}^{a}\left(i_{s}\right) t_{a}^{0}, \\
& c_{a}^{s}\left(i_{s}\right)=g_{c}^{a}\left(i_{s}\right) c_{a}^{0},
\end{aligned}
$$

are the free-flow travel time and capacity of link $a$ under scenario $s$ (intensity $i_{s}$ ), respectively; $t_{a}^{0}$ and $c_{a}^{0}$ denote free-flow travel time and capacity of link $a$ under good weather scenario $\left(i_{s}=0\right)$, respectively; while $g_{t}^{a}\left(i_{s}\right)$ and $g_{c}^{a}\left(i_{s}\right)$ are the scaling functions of scenario $s$ (intensity $i_{s}$ ) on free-flow travel time and ideal link capacity under good weather scenario, respectively. The heavier the adverse weather intensity (i.e., a larger $i_{s}$ ), the higher the free-flow travel time and the lower link capacity. Therefore, the scaling functions should hold the following features [13]:

(a) $g_{t}^{a}\left(i_{s}\right)$ is an increasing function with respect to $i_{s}$ indicating that the free-flow travel time increases with the adverse weather intensity.

(b) $g_{c}^{a}\left(i_{s}\right)$ is a decreasing function with respect to $i_{s}$ indicating that the link capacity decreases with the adverse weather intensity. (c) $g_{t}^{a}(0)=g_{c}^{a}(0)=1$ indicates that when the weather is clear $\left(i_{s}=0\right)$, this link travel time function (1) is equivalent to the conventional BPR function.

The actual travel time of route $r$ between O-D pair $w$ in scenario $s$ (intensity $i_{s}$ ) is:

$$
t_{r w}^{s}=\sum_{a \in A} \delta_{a r w} t_{a}^{s}\left(i_{s}, x_{a}\right) \quad \forall s \in S, \quad r \in R_{w}, w \in W .
$$

3.2. Effects of Information from Weather Forecast on TBI. Weather forecast usually is a familiar and useful information source, based on which travelers can make their individual travel arrangements to maximize their expected utilities. Travelers can acquire real-time weather forecast information via many approaches, e.g., vehicle-mounted radio broadcasting programs or weather forecast apps, etc. Compared with TPI who can acquire actual route travel time of the network, TBI without ATIS in this study are assumed to have the identical attitude to a piece of weather forecast information. However, weather forecast results cannot always perfectly match the physical truth, which is known as its prediction bias. Therefore, besides the information about adverse weather intensity $i_{s}$ under adverse weather scenario $s$, weather forecast also issue corresponding predictive probability $P_{s}$, e.g., heavy rainfall $\left(i_{s}=30 \mathrm{~mm} / \mathrm{h}\right)$ with the occurring probability of $80 \%\left(P_{3}\right)$.

Let $P=\left\{\widetilde{p}_{1}, \breve{p}_{2}, \cdots, \widetilde{p}_{s}, \cdots, \widetilde{p}_{|S|}\right\}$ represent the set of prior occurring probability of each network scenario $s$ from the weather forecast. For instant $P=\{5 \%, 10 \%, 70 \%, 10 \%, 5 \%\}$ means that, the weather forecast predicts that light rain, moderate rain, heavy rain, rainstorm and heavy rainstorm would occur tomorrow with the chances of $5 \%, 10 \%, 70 \%, 10 \%$ and $5 \%$, respectively. Then following Lam et al. [13] and based on Bayes' formula, we can obtain that $\bar{p}^{s}$, the perceived occurring probability of scenario $s$ of TBI under weather forecasting probability set $P$, is:

$$
\bar{p}_{s / \bar{p}_{s}}^{s}=\frac{d_{\widetilde{p}_{s} / s} \widetilde{p}_{s}}{\sum_{s=1}^{|S|} d_{\widetilde{p}_{s} / s} \widetilde{p}_{s}} \quad \forall s \in S,
$$

in which $d_{\widetilde{p}_{s} / s}$ is the perceived conditional probability of $\widetilde{p}_{s}$ given scenario $s$ which represents the perceived average accuracy of the weather forecast according to the previous experience of a TBI. For example, $d_{\vec{p}_{s} / s}=d_{0.7 / 3}=0.8$ means that based on the previous experience of an arbitrary TBI, when a heavy rain $(s=3)$ occurred, this traveler estimates that the average prediction probability that the weather forecast had predicted $p_{3}=0.7$ is 0.8 , which hints that he/she considers that the forecast information is acceptably accurate. The subscript set $\{1,2, \cdots, s, \cdots,|S|\}$ consists of all $|S|$ adverse weather scenarios of this adverse weather condition. For occurring probability of the weather scenario $s$ perceived by any TBI, the conservation equation holds:

$$
\sum_{s=1}^{|S|} \hat{p}^{s}=1 \quad \forall s \in S
$$


3.3. Basic Model and Its Characteristics. Let $x_{a 1}^{s}$ and $x_{a 2}^{s}$ denote the flow of TPI and TBI under scenario $s \in S$ on link $a$, respectively. Assume that $t_{a 1}^{s}$ and $t_{a 2}$ denote the perceived link travel time of TPI under scenario $s$ and TBI on link $a$, respectively. This study assumes that the following formulas are tenable:

$$
t_{a j}^{s}=t_{a j}^{s}\left(x_{a}^{s}, i_{s}\right)=t_{a j}^{s}\left(x_{a 1}^{s}, x_{a 2}^{s}, i_{s}\right) \quad \forall a \in A, s \in S, j=1,2,
$$

in which

$$
x_{a}^{s}=\varphi x_{a 1}^{s}+\gamma x_{a 2}^{s} \quad \varphi, \gamma>0, \quad \forall a \in A, s \in S .
$$

To be specific, formula (7) assumes that the cost function $t_{a j}$ of link $a$ merely correlates with the flow of $j$ categories of travelers (i.e., TPI and TBI) on link $a$, and is independent of flows on other links, e.g., $x_{b j}, \forall b \in A, b \neq a, j=1,2$. We further assume that the total equivalent flow on link $a$ is a linear combination of TPI flow $x_{a 1}^{s}$ and TBI flow $x_{a 2}^{s}$ on this link, with weighting coefficients being $\varphi, \gamma>0$, as formulated in formula (8). For example, if the flows of TPI and TBI have even contributions (weight) on travel time of link $a$, (i.e., $\varphi=\gamma=1$ ), the total flow of link $a$ just equals to the flow summation of TPI and TBI, i.e.,

$$
x_{a}^{s}=x_{a 1}^{s}+x_{a 2}^{s} \quad \forall a \in A, \forall s \in S,
$$

then formula (7) turns to be

$$
t_{a j}^{s}=t_{a j}^{s}\left(x_{a 1}^{s}+x_{a 2}^{s}, i_{s}\right) \quad \forall a \in A, \quad s \in S, \quad j=1,2 .
$$

For TPI, their perceived link travel time $t_{a 1}^{s}$ is the actual travel time $t_{a}^{s}$ of link $a$ in scenario $s$ (intensity $i_{s}$ ) acquired from ATIS, shown as (11). While for TBI, their perceived link travel time $t_{a 2}$ is the perceived synthetic link travel time combining their personal perception on the accuracy of weather forecast information with kinds of actual link travel time in all scenarios $|S|$, shown as formula (12):

$$
\begin{aligned}
t_{a 1}^{s}=t_{a}^{s}\left(i_{s}, \mathbf{x}_{a}\right) & =t_{a}^{s}\left(x_{a 1}^{s}, x_{a 2}^{s}, i_{s}\right) \quad \forall s \in S, \quad a \in A, \quad(11) \\
t_{a 2}=\sum_{s} \widehat{p} t_{a}^{s}\left(i_{s}, \mathbf{x}_{a}\right) & =\sum_{s} \widehat{p} t_{a}^{s}\left(x_{a 1}^{s}, x_{a 2}^{s}, i_{s}\right) \quad \forall s \in S, a \in A .
\end{aligned}
$$

To make the problem we studied herein prominent and clearly, we assume that the travel demand under a certain network scenario is deterministic. Assume that $d_{1 w}^{s}$ and $d_{2 w}^{s}$ denote the travel demand of TPI and TBI traveling between OD pair $w$ and under adverse weather scenario $s$, respectively. Then the flow conservation constraint equations can be formulated as

$$
\begin{gathered}
d_{j w}^{s}=\sum_{r \in R_{w}} f_{j r w}^{s} \quad \forall r \in R_{w}, \quad w \in W, \quad s \in S, j=1,2, \\
f_{j r w}^{s} \geq 0 \quad \forall s \in S, \quad r \in R_{w}, w \in W, j=1,2, \\
x_{a j}^{s}=\sum_{w} \sum_{r} \delta_{\text {arw }} f_{j r w}^{s} \quad \forall a \in A, \quad r \in R_{w}, \quad w \in W, \quad s \in S, \quad j=1,2,
\end{gathered}
$$

TABLE 2: The completed information-knowledge matrix of travel demand.

\begin{tabular}{lc}
\hline Network Information Levels (NILs) & \\
\hline TPI $(j=1)$ & TBI $(j=2)$ \\
$\left(N_{1 w}, P_{1 w}\right)$ & $\left(N_{2 w}, P_{2 w}\right)$ \\
\hline
\end{tabular}

which jointly form the feasible region of link flow pattern $\mathbf{x}=\left[x_{\text {jarw }}\right]_{|S||A||J|}$ in which $|J|=2$.

The route choice criteria of TPI-Actual Route Travel Time (ARTT). Given a specific adverse weather scenario $s$, the perceived route travel time of TPI on route $r$ connecting OD pair $w$, i.e., ARTT- $t_{1 r w}^{s}$, can be written as

$t_{1 r w}^{s}=t_{r w}^{s}=\sum_{a} \delta_{a r w} t_{a}^{s}\left(i_{s}, x_{a 1}^{s}, x_{a 2}^{s}\right) \quad \forall s \in S, \quad r \in R_{w}, w \in W$.

The route choice criteria of TBI-Perceived Route Travel Time (PRTT). Given all AWSs $s \in S$ that might occur, the perceived route travel time of TBI on route $r$ connecting OD pair $w$, i.e., PRTT- $t_{2 r w}$, can be written as

$t_{2 r w}=\sum_{s} \tilde{p}^{s} t_{r w}^{s}=\sum_{s} \tilde{p}^{s} \sum_{a} \delta_{a r w} t_{a}^{s}\left(i_{s}, x_{a 1}^{s}, x_{a 2}^{s}\right) \quad \forall r \in R_{w}, \quad w \in W$,

in which $\widehat{P}^{s}$ is the perceived occurring probability of TBI under scenario $s$ given in (5).

Assume that $N_{w}, w \in W$ denotes the total travel demand between OD pair $w$, then $N_{w}$ consists of TPI and TBI. As shown in Table 2, $N_{1 w}$ denotes the quantity of TPI, and $P_{1 w}$ indicates corresponding proportion, then we have $N_{1 w}=N_{w} P_{1 w}$. Analogously, $N_{2 w}$ and $P_{2 w}$ denote the quantity and proportion of TBI, then we also have $N_{2 w}=N_{w} P_{2 w}$, $N_{1 w}+N_{2 w}=N_{w}, P_{1 w}+P_{2 w}=1$.

3.4. Equilibrium Conditions. (1) Any TPI chooses a route with the minimum ARTT $\left(\zeta_{1 w}^{s *}\right)$ given network scenario $s$. So the following equilibrium conditions must hold for TPI traveling on all routes between O-D pair $w$ under an arbitrary network scenario $s$ :

$$
t_{1 r w}^{s}\left(\tilde{\boldsymbol{x}}^{*}\right)\left\{\begin{array}{l}
=\zeta_{1 w}^{s *}, \text { if } f_{1 w}^{s *} \geq 0 \\
\geq \zeta_{1 w}^{s *}, \text { if } f_{1 r w}^{s *}=0
\end{array} \quad \forall r \in R_{w}, w \in W, s \in S .\right.
$$

For all TPI who travel between OD pair $w$, no one can further lower his ARTT by unilaterally altering his travelling route; the ARTT of all utilized routes between OD pair $w$ are equal and minimal $\left(\zeta_{1 w}^{s *}\right)$, and will not larger than that of any unutilized routes.

(2) As discussed above, any TBI chooses a route with the minimum PRTT $\left(\zeta_{2 w}^{s *}\right)$ given all network scenarios. So the following equilibrium conditions must hold for TBI of knowledge level $k$ traveling on all routes between O-D pair $w$ under any network scenario.

$$
t_{2 r w}\left(\tilde{\boldsymbol{x}}^{*}\right)\left\{\begin{array}{l}
=\zeta_{2 w}^{*}, \text { if } f_{2 r w}^{*} \geq 0 \\
\geq \zeta_{2 w}^{*}, \text { if } f_{2 r w}^{*}=0
\end{array} \forall r \in R_{w}, w \in W .\right.
$$


No matter how the network scenario $s \in S$ would be, for any TBI traveling between OD pair $w$, no one can further lower his PRTT by unilaterally altering his travelling route; the PRTT of all utilized routes between OD pair $w$ are equal and minimal $\left(\zeta_{2 w}^{*}\right)$, and will not larger than that of any unutilized routes.

The travel demands of both TPI and TBI between OD pair $w$ satisfy the flow conservation constraint Equations (13)-(15).

3.5. A Variational Inequality Formulation. The nonlinear complementarity conditions can be formulated as a finitedimentional variational inequality (VI) problem:

$$
\left\langle\mathbf{t}\left(\mathbf{x}^{*}\right)^{T}, \mathbf{x}-\mathbf{x}^{*}\right\rangle \geq 0, \quad \forall \mathbf{x} \in \mathbf{X} .
$$

in which, $\mathbf{t}\left(\mathbf{x}^{*}\right)=\left[t_{\text {jarw }}\right]_{|S||A||J|}, \mathbf{x}=\left[x_{\text {jarw }}\right]_{|S||A||J|},\langle\cdot, \cdot\rangle$ denotes $|S||A||J|$-dimentional Euclidean space. $\mathbf{X}$ is the feasible region of $\mathbf{x} \cdot \mathbf{x}^{*}$ is the matrix of optimal solution of $\mathbf{x}=\left[x_{\text {jarw }}\right]_{|S||A||J|}$. Equation (20) can be also reformulated as

$$
\sum_{s} \sum_{a} t_{a 1}^{s *}\left(x_{a 1}^{s}-x_{a 1}^{s *}\right)+\sum_{s} \sum_{a} t_{a 2}^{*}\left(x_{a 2}^{s}-x_{a 2}^{s *}\right) \geq 0 .
$$

Theorem 1. Given adverse weather scenario $s \in S$, the twoclass user link flow pattern $\mathbf{x}=\left[x_{\text {jarw }}\right]_{|S||A||J|} \in \mathbf{X}$ that consists of TPI and TBI accords with the equilibrium solution, i.e., satisfying the equilibrium conditions (18) and (19), if and only if it satisfies the VI problem (20).

\section{Proof. See Appendix.}

As with the existence and uniqueness of the equilibrium solution, though inserted by scaling functions that can measure adverse weather intensities, the GBPR function is still continuous in the feasible region $\mathbf{X}$. Besides, the feasible region $\mathbf{X}$ is a typical non-empty compact set and convex set. Therefore, at least one solution $\mathbf{x}^{*}$ of the VI problem (20) exists [17]. Given congestion effects, the GBPR function in this study is a continuous, strictly monotonic increasing function of link flow, and the feasible region $\mathbf{X}$ is a typical non-empty compact set and convex set. Thus, the solution of VI problem (20) is unique [17].

\section{Solution Algorithm}

The stochastic network user equilibrium problem given route choice heterogeneity of travelers is a typical multi-class, asymmetric network equilibrium problem. Though the Jacobian of link cost function in this problem is asymmetric, the precondition still requires that the Jacobian is positive definite. Therefore, the Streamlined Diagonalization Algorithm (SDA) [18], is adopted to solve the proposed asymmetric SN-UE VI problem. The solution generated by each iteration is input as the initial solution of next iteration. The SDA for solving asymmetric network UE problem consists of the following general steps:

Step 0. Network initialization. Set $n:=0$. Find a feasible link-flow pattern vector $\mathbf{x}^{n}$;
Step 1. Link travel time updating. Set $t_{a}^{n}=t_{a}\left(\mathbf{x}^{n}\right), \quad \forall a \in A$.

Step 2. Direction finding. Assign the O-D flows, $\left\{q^{w}\right\}$, to the network utilizing all-or-nothing approach based on $\left\{t_{a}^{n}\right\}$, which yields a link-flow pattern; $\left\{y_{a}^{n}\right\}$ :

Step 3. Move-size determination. Find a scalar, $\lambda_{n}$, which solves the following program:

$$
\begin{gathered}
\min z\left(\lambda_{n}\right)=\sum_{a \in A} \int_{0}^{x_{a}^{n}+\lambda_{n}\left(y_{a}^{n}-x_{a}^{n}\right)} t_{a}\left(x_{1}^{n}, \ldots, x_{a-1}^{n}, \omega, x_{a+1}^{n}, \ldots, x_{A}^{n}\right) \mathrm{d} \omega \\
\text { s.t. } 0 \leq \lambda_{n} \leq 1 .
\end{gathered}
$$

Step 4. Link-flow updating. Set $x_{a}^{n+1}=x_{a}^{n}+\lambda_{n}\left(y_{a}^{n}-x_{a}^{n}\right), \forall a \in A$;

Step 5. Convergence test. If $x_{a}^{n+1} \approx x_{a}^{n}, \forall a$, stop. The solution is $\left\{x_{a}^{n+1}\right\}$; otherwise, set $n=n+1$ and go to Step 1 .

SDA is similar to the Frank-Wolfe algorithm [19] that solves the traditional UE problem, which mainly differs in that the SDA's update of each link travel time is based on the whole network flow, i.e., $t_{a}^{n}=t_{a}\left(\mathbf{x}^{n}\right)$, rather than $t_{a}^{n}=t_{a}\left(x_{a}^{n}\right)$ in F-W algorithm. The diagonalization principle reflects in that, in the iteration process of step size $\lambda_{n}$, the cost function of each link $a$ is merely related to its own link flow $x_{a}^{n}$, while the flows of other links are fixed in $\mathbf{x}^{n}$. With respect to the convergence of SDA, please see reference [20].

\section{Numerical Examples}

In this section, a small-size triangle network and a middle-size Sioux Falls network are used to testify the validity of the model and solution algorithm proposed in this study. To show the efficiency of our model and algorithm, we test our examples on a PC with a $3.40 \mathrm{GHz}$ CPU, an $8 \mathrm{~GB}$ RAM and Windows 10 Enterprise 64-bit operating system. The model was coded with Matlab.

\subsection{A Small-Size Triangle Network}

5.1.1. Network Parameter Settings and Descriptions. A small triangle network consisting of 4 nodes, 5 links (denoted in italics), 2 O-D pairs and 4 routes is presented in Figure 1.

The link-route incidence matrix is shown in Table 3 . The functional form and parameters of the scaling functions $g_{t}^{a}\left(i_{s}\right)$ and $g_{c}^{a}\left(i_{s}\right)$ are shown in Table 4 .

Four network scenarios $s$, i.e., small rain $(s=1)$, moderate rain $(s=2)$, heavy rain $(s=3)$, rainstorm $(s=4)$ and corresponding rainfall intensities $i_{s}$ are shown in Table 5, respectively.

The perceived conditional probability distribution of the TBI is listed in Table 6.

This example employs the following form of link cost function under adverse weather conditions:

$$
t_{a}^{s}\left(i_{s}, x_{a}\right)=g_{t}^{a}\left(i_{s}\right) t_{a}^{0}+\alpha\left(\frac{x_{a}}{g_{c}^{a}\left(i_{s}\right) c_{a}^{0}}\right)^{\beta} \quad \forall a \in A, \quad s \in S .
$$

in which $\alpha=0.15, \beta=4.0$. 


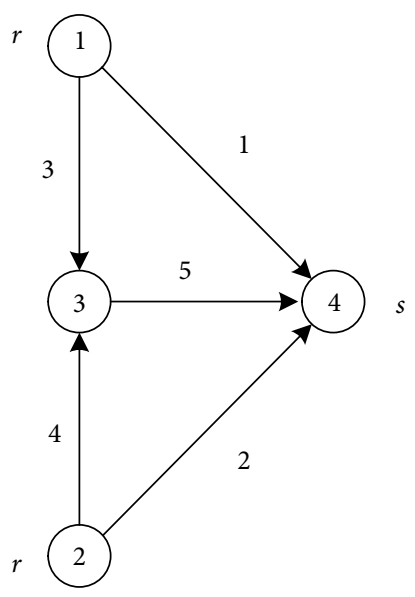

Figure 1: A small size triangle network.

TABLE 3: Route-link incidence.

\begin{tabular}{lccc}
\hline Route & O-D pair $(r-s)$ & Link chain & Node chain \\
\hline R1 & \multirow{2}{*}{$1-4$} & 1 & $1-4$ \\
R2 & & $3-5$ & $1-3-4$ \\
R3 & $2-4$ & $4-5$ & $2-3-4$ \\
R4 & & 2 & $2-4$ \\
\hline
\end{tabular}

TABLE 4: Network configuration inputs.

\begin{tabular}{lccccc}
\hline Parameters & \multicolumn{5}{c}{ Link $a$} \\
& 1 & 2 & 3 & 4 & 5 \\
\hline$t_{a}^{0}(\mathrm{~h})$ & $2 / 3$ & $1 / 2$ & $1 / 4$ & $1 / 3$ & $1 / 3$ \\
$c_{a}^{0}(\mathrm{pcu} / \mathrm{h})$ & 1600 & 1600 & 1300 & 1200 & 2000 \\
$g_{t}^{a}\left(i_{s}\right)$ & $g_{t}^{a}\left(i_{s}\right)=\exp \left(0.05 i_{s}\right)$ & $g_{t}^{a}\left(i_{s}\right)=\exp \left(0.07 i_{s}\right)$ \\
$g_{c}^{a}\left(i_{s}\right)$ & $g_{c}^{a}\left(i_{s}\right)=\exp \left(-0.02 i_{s}\right)$ & $g_{c}^{a}\left(i_{s}\right)=\exp \left(-0.04 i_{s}\right)$ \\
Link types & Weather-resistant links & Weather-sensitive links \\
\hline
\end{tabular}

As shown in Table 7, the total traffic demands between OD pairs 1-4, 2-4 are set to be 1500 and 2000 units, respectively. The proportion of TPI and TBI between OD pair 1-4 are set to be $30 \%$ and $70 \%$, respectively, while these proportions between OD pair $2-4$ are $60 \%$ and $40 \%$, respectively. OD pair 1-4 and 2-4 can be considered as the developing and developed regions, respectively, for the following reasons: (1) the penetration rate of ATIS in developed regions is usually higher, so is the proportion of the TPI $(60 \%>30 \%)$; (2) the social and economic activity would be more active in the developed region $(2000>1500)$, so more traffic demand is expected.

The convergence performances (the total iteration $n^{*}$ and convergence gap $e^{*}$ ) of the six cases considered in this example (i.e., Z1-Z6) are listed in Table 8.

Take case Z1, i.e., small rain $\left(s=1, i_{s}=2.5\right)$, as an example, the algorithm converges at iteration $n=110$ and the gap $e=\max _{a}\left\{\left|\left(x_{a}^{n+1}-x_{a}^{n}\right) / x_{a}^{n}\right|\right\} \times 100 \%=0.0099<\varepsilon=0.01$ The blue curve and the red curve in Figure 2 denote the iteration step size $\lambda_{n}$ and the convergence gap $e_{n}$, respectively. As shown
TABLE 5: Rainfall network scenarios and corresponding intensities.

\begin{tabular}{lc}
\hline Network scenarios $s$ & Rainfall intensity $i_{s}(\mathrm{~mm} / \mathrm{h})$ \\
\hline Small rain $(s=1)$ & 2.5 \\
Moderate rain $(s=2)$ & 8 \\
Heavy rain $(s=3)$ & 15 \\
Rainstorm $(s=4)$ & 30 \\
\hline
\end{tabular}

TABLE 6: Different occurring probability (OP) and conditional probability distribution of TBI.

\begin{tabular}{|c|c|c|c|c|c|c|c|c|}
\hline \multirow[t]{2}{*}{ Cases } & \multicolumn{4}{|c|}{$\begin{array}{l}\text { OP from weather } \\
\text { forecast: } p_{s}(\%)\end{array}$} & \multicolumn{4}{|c|}{$\begin{array}{l}\text { Conditional probability of } \\
p_{s}: d_{p_{s} / s}(\%)\end{array}$} \\
\hline & $\breve{p}_{1}$ & $\widetilde{p}_{2}$ & $\widetilde{p}_{3}$ & $\bar{p}_{4}$ & $d_{\vec{p}_{1} / 1}^{1}$ & $d_{\vec{p}_{2} / 2}^{1}$ & $d_{\vec{p}_{3} / 3}^{1}$ & $d_{\bar{p}_{4} / 4}^{1}$ \\
\hline $\mathrm{Z1}$ & 80 & 10 & 6 & 4 & 90 & 5 & 3 & 2 \\
\hline $\mathrm{Z} 2$ & 9 & 75 & 9 & 7 & 7 & 80 & 8 & 5 \\
\hline $\mathrm{Z3}$ & 8 & 12 & 70 & 10 & 8 & 12 & 70 & 10 \\
\hline $\mathrm{Z4}$ & 7 & 10 & 18 & 65 & 9 & 12 & 19 & 60 \\
\hline $\mathrm{Z5}$ & 1 & 4 & 90 & 5 & 1 & 4 & 90 & 5 \\
\hline Z6 & 1 & 4 & 90 & 5 & 25 & 25 & 25 & 25 \\
\hline
\end{tabular}

TABLE 7: Proportion of TBI and TPI in traffic demand in this case.

\begin{tabular}{|c|c|c|c|c|}
\hline $\begin{array}{l}\text { O-D pair } \\
(r-s)\end{array}$ & $\begin{array}{c}\text { Network } \\
\text { areas }\end{array}$ & TPI (\%) & TBI (\%) & $\begin{array}{c}\text { Total } \\
\text { demand }\end{array}$ \\
\hline $1-4$ & $\begin{array}{l}\text { Develop- } \\
\text { ing area }\end{array}$ & $450(30 \%)$ & $\begin{array}{c}1050 \\
(70 \%)\end{array}$ & 1500 \\
\hline $2-4$ & $\begin{array}{c}\text { Developed } \\
\text { area }\end{array}$ & $\begin{array}{c}1200 \\
(60 \%)\end{array}$ & $800(40 \%)$ & 2000 \\
\hline \multicolumn{2}{|c|}{ In total } & 1650 & 1850 & 3500 \\
\hline
\end{tabular}

in this figure, the values of $\lambda_{n}$ and $e$ reach to a relatively steady level at iteration $n=20,\left(\lambda_{20}=0.0312, e_{20}=0.0332\right)$, but the convergence gap $e$ does not satisfy the convergence criterion $(\varepsilon=0.01)$ until another 90 iterations (i.e., $n=110)$, which implies that the SDA converges rapidly in the forepart of the iteration, but may perform tardily in the latter stage.

5.1.2. Optimal Solution (Z1). The optimal solution of link flows in $\mathrm{Z1}\left(s=1\right.$, small rain, $\left.i_{1}=2.5\right)$ is

$$
\mathbf{x}^{*}=\left[\begin{array}{cc}
x_{11}^{*} & x_{12}^{*} \\
x_{21}^{*} & x_{22}^{*} \\
x_{31}^{*} & x_{32}^{*} \\
x_{41}^{*} & x_{42}^{*} \\
x_{51}^{*} & x_{51}^{*}
\end{array}\right]=\left[\begin{array}{rc}
56.73 & 614.55 \\
960.21 & 766.63 \\
393.27 & 435.45 \\
239.79 & 33.37 \\
633.06 & 468.81
\end{array}\right]
$$

and is further depicted in Figure 3, in which the blue and red numbers marked beside a link denote the flow of TPI and TBI on that link, respectively. According to formula (9), the equilibrium solution of the total link flows (denoted as black numbers in the links) is:

$$
\begin{aligned}
\mathbf{x}_{a}^{*} & =\left[\begin{array}{lllll}
x_{1}^{*} & x_{2}^{*} & x_{3}^{*} & x_{4}^{*} & x_{5}^{*}
\end{array}\right]^{T} \\
& =\left[\begin{array}{llllll}
671.28 & 1726.85 & 828.72 & 273.15 & 1101.88
\end{array}\right]^{T} .
\end{aligned}
$$


TABLE 8: Summary of iterations of the SDA.

\begin{tabular}{lcc}
\hline Cases & Iterations $n^{*}$ & $e^{*}=\max _{a}\left\{\left|\left(x_{a}^{n+1}-x_{a}^{n}\right) / x_{a}^{n}\right|\right\} \times 100 \%<\varepsilon=0.01$ \\
\hline $\mathrm{Z} 1$ & 110 & 0.0099 \\
$\mathrm{Z} 2$ & 77 & 0.0072 \\
$\mathrm{Z3}$ & 66 & 0.0054 \\
$\mathrm{Z} 4$ & 46 & 0.0063 \\
$\mathrm{Z5}$ & 93 & 0.0022 \\
$\mathrm{Z6}$ & 121 & 0.0091 \\
\hline
\end{tabular}

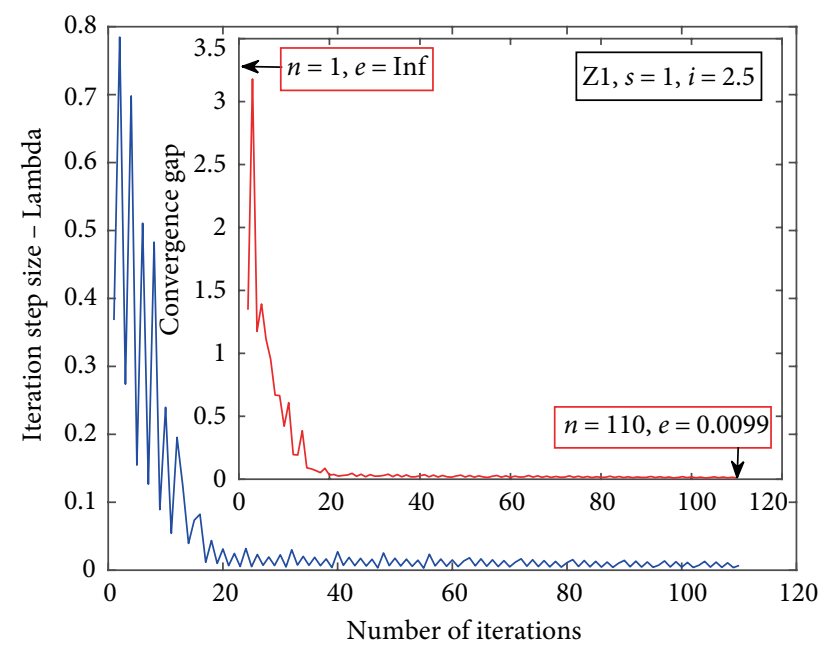

FIGURE 2: Curve of convergence gap of SDA in this example.

For Figure 3, the proportion (80.02\%) of TPI in developed area (O-D pair 2-4) choosing route $\mathrm{R} 4$ (i.e., link 2) is higher than that $(12.6 \%)$ of TPI in developing area (O-D pair 1-4) choosing route $\mathrm{R} 1$ (i.e., link 1 ). This result is expected because the ATIS penetration rate of the developed areas $(60 \%)$ is higher than that of the developing areas (30\%). When the network has not been heavily worsened by the rainfall (small rain), more travelers in developed areas can capture actual route travel time of the network and then are likely to prefer route R4, which is a high-capacity, "weather-resistant" route compared with R3. Analogously, given that the proportion (70\%) of the TBI who are not armed with ATIS in developing area (O-D pair 1-4) is higher than that (40\%) of those in developed area (O-D pair 2-4), in the equilibrium condition, more TBI (41.47\%) in developing area (O-D pair 1-4) select route R2 (i.e., link 3-5) than those TBI (4.17\%) in developed area (O-D pair 2-4) who select R3 (i.e., link 4-5).

On the other hand, we can learn from Figure 3 that, for the proportion of TPI is higher in the developed area (O-D pair $2-4)$, more travelers (86.34\%) prefer to "weather-resistant" route $\mathrm{R} 4$ than that of travelers (44.75\%) in the developing area (O-D pair 1-4) who select the "weather-resistant" route R1. This result suggests that ATIS makes more TPI turn to high-capacity, "weather-resistant" routes with more probability being a shorter route travel time. While without the help of ATIS, travelers (i.e., TBI) who make their route choices according to their existing travel experience will spread evenly on the "weather-resistant" and "weather-sensitive" routes in the whole network.

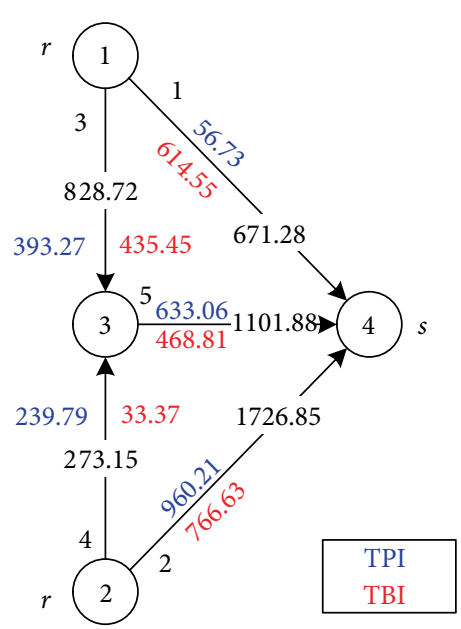

FIgURE 3: Optimal solutions of traffic assignment in case Z1 $(s=1$, $\left.i_{1}=2.5\right)$.

The iteration-convergence procedures of the link flows of TPI, TBI and the total link flow (TPI+TBI) are presented in Figures 4(a) and 4(b) and Figure 5, respectively.

According to the optimal solution $\mathbf{x}^{*}$ of the link flow, the perceived route travel time of TPI and TBI are:

$$
\begin{aligned}
\boldsymbol{t}^{*} & =\left[\begin{array}{ll}
\boldsymbol{t}_{1 r(1-4)}^{s=1^{*}} & \boldsymbol{t}_{1 r(2-4)}^{s=1^{*}} \\
\boldsymbol{t}_{2 r(1-4)}^{s=1^{*}} & \boldsymbol{t}_{2 r(2-4)}^{s=1^{*}}
\end{array}\right] \\
& =\left[\begin{array}{ccccc}
0.7611 & 0.7525 & \vdots & 0.8154 & 0.8152 \\
--- & --- & --- & ---- & --- \\
0.7669 & 0.7681 & \vdots & 0.8282 & 0.8237
\end{array}\right] \quad r=1,2
\end{aligned}
$$

in which the elements in the first and second rows are the ARTT of TPI and the PRTT of TBI between the two OD pairs, respectively. Accordingly, the corresponding iteration-convergence procedures are depicted in Figures 6(a) and 6(b), respectively. According to the route travel time in the equilibrium state of this example (formula (26), Figures 6(a) and 6(b)), no matter which class a traveler belongs to, the route travel time in developed area (O-D pair 2-4) (i.e., 0.81 and 0.82 for TPI and TBI, respectively) is higher than that in developing area (O-D pair 1-4) (about 0.76 ), which results from the following reasons: (1) the traffic demand (2000) in developed area (O-D pair 2-4) is higher than that (1500) in developing area (O-D pair 1-4); (2) high ATIS penetration rate in developed area intensifies the travelers' aggregation effects on "weather-resistant" routes to seek for lower travel time cost under adverse weather conditions. 


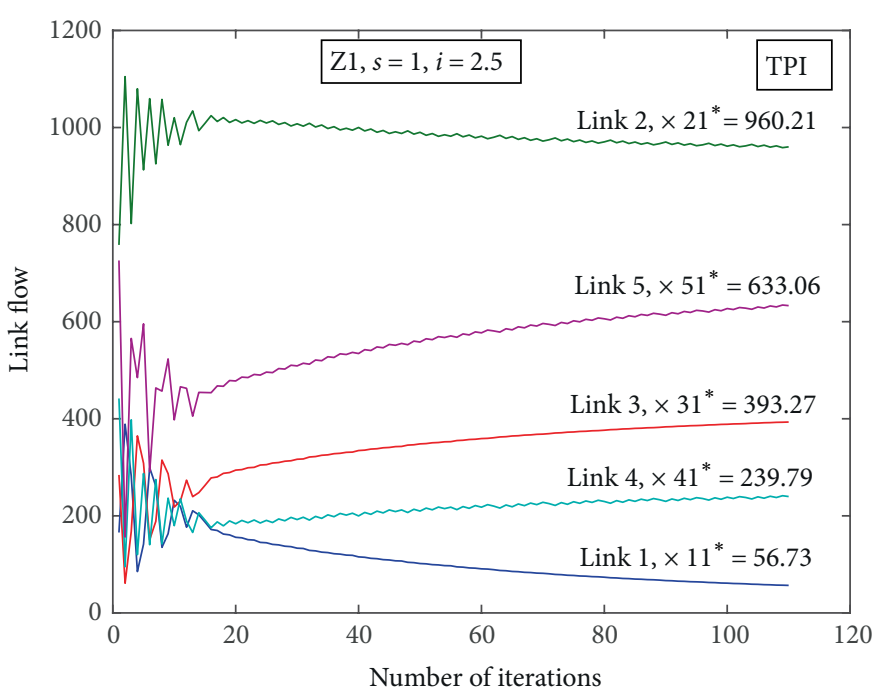

(a)

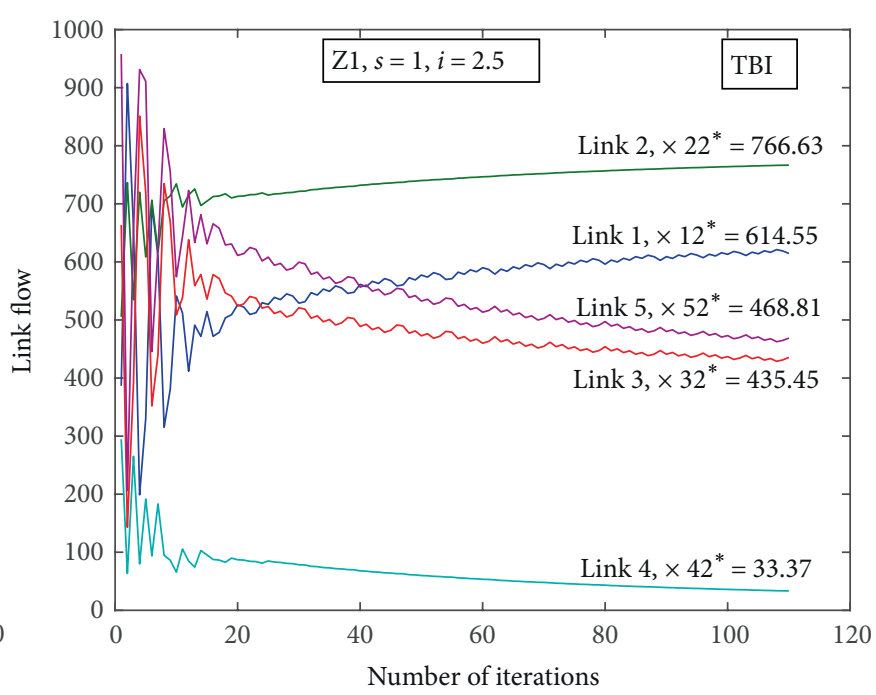

(b)

FIGURE 4: Convergence curves of link flows of TPI or TBI $(Z 1, s=1)$. (a) Convergence curves of link flows of TPI $(\mathrm{Z} 1, s=1)$. (b) Convergence curves of link flows of TBI $(\mathrm{Z} 1, s=1)$.

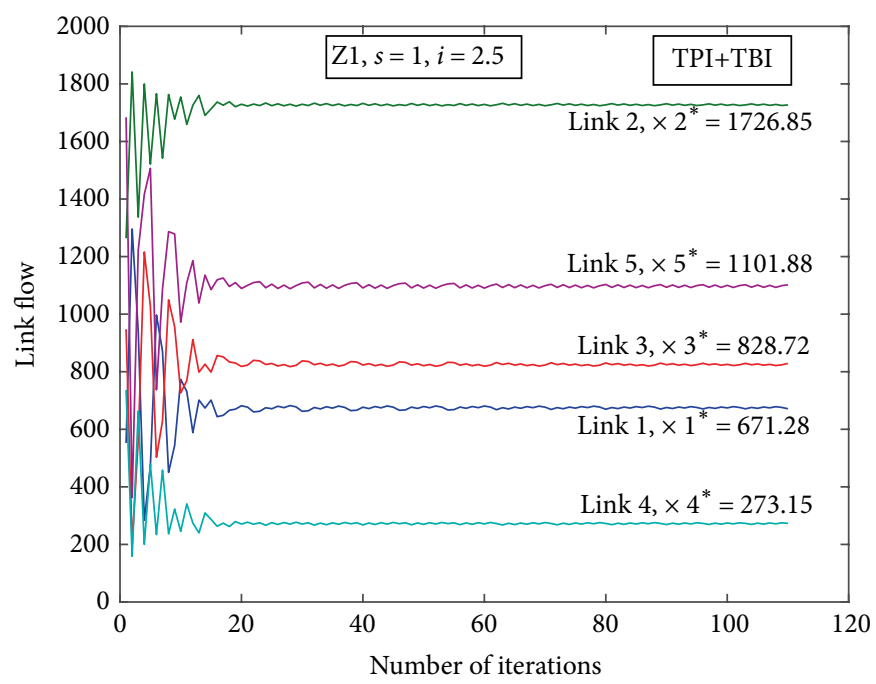

FIGURE 5: Convergence curves of total link flows of TPI \& TBI $(\mathrm{Z} 1, s=1)$.

Moreover, we can learn from (26) that, in the case $\mathrm{Z1}$ (small rain), the PRTT of TBI are always larger than the ARTT of TPI who use that route (i.e., $\mathbf{t}_{2 r w}^{s=1 *}>\mathbf{t}_{1 r w}^{s=1 *}$ ), which could be proved in mathematics.

The convergence curves of ARTT of TPI (Figure 6(a)) show that, the sub-network of the developed area (i.e., R3 \& $\mathrm{R} 4$ between $\mathrm{O}-\mathrm{D}$ pair 2-4) reaches to equilibrium state rapidly within 20 iterations. By contrast, the sub-network of the developing area (i.e., R1 \& R2 between O-D pair 1-4) reaches to an approximate equilibrium state with the travel time differential of the two routes is less than $1.2 \%$. Analogous results can be also observed from the convergence curves of PRTT of TBI (Figure 6(b)) that, the sub-network of the developing area (i.e., $\mathrm{R} 1$ \& R2 between O-D pair 1-4) reaches to equilibrium state rapidly, while the sub-network of the developed area (i.e., R3 $\& \mathrm{R} 4$ between $\mathrm{O}-\mathrm{D}$ pair $2-4$ ) reaches to an approximate equilibrium state with the travel time differential of the two routes is about $0.55 \%$. These derive from the fact that the SDA might contribute limitedly to the further improvement of the objective functions when it is not highly demanding on the solution precision.

\subsubsection{Equilibrium Solutions of the Link Flow of the TPI and TBI}

in Cases Z1-Z4. The equilibrium solutions in scenario Z1-Z4 are presented in Figures $7(\mathrm{a})-7(\mathrm{~d})$. From the prospective of the total link flow, with the deteriorating of rainfall scenarios with the highest occurring probability moving from small rain $(\mathrm{Z1}, 80 \%)$ to rainstorm $(\mathrm{Z} 4,65 \%)$, more and more travelers in the developed area (i.e., O-D pair 2-4) move from "weathersensitive" route R3 to the "weather-resistant" route R4 (17271773 - 1827-1885, the total traffic demand is 2000). Similar results can be also observed in the developing area (i.e., O-D 


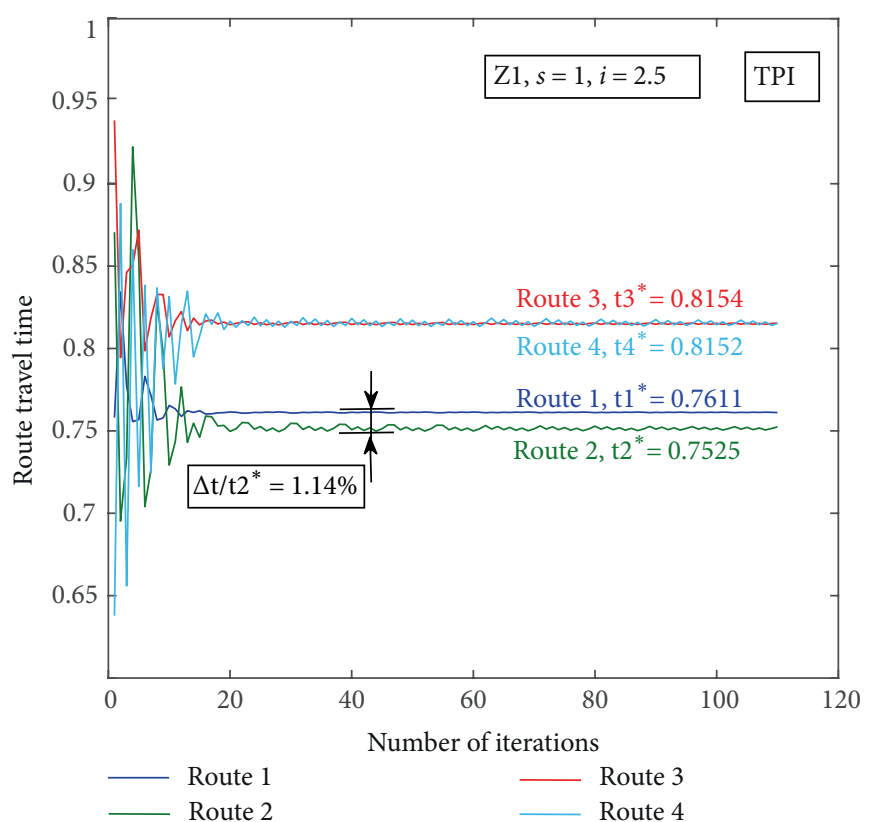

(a)

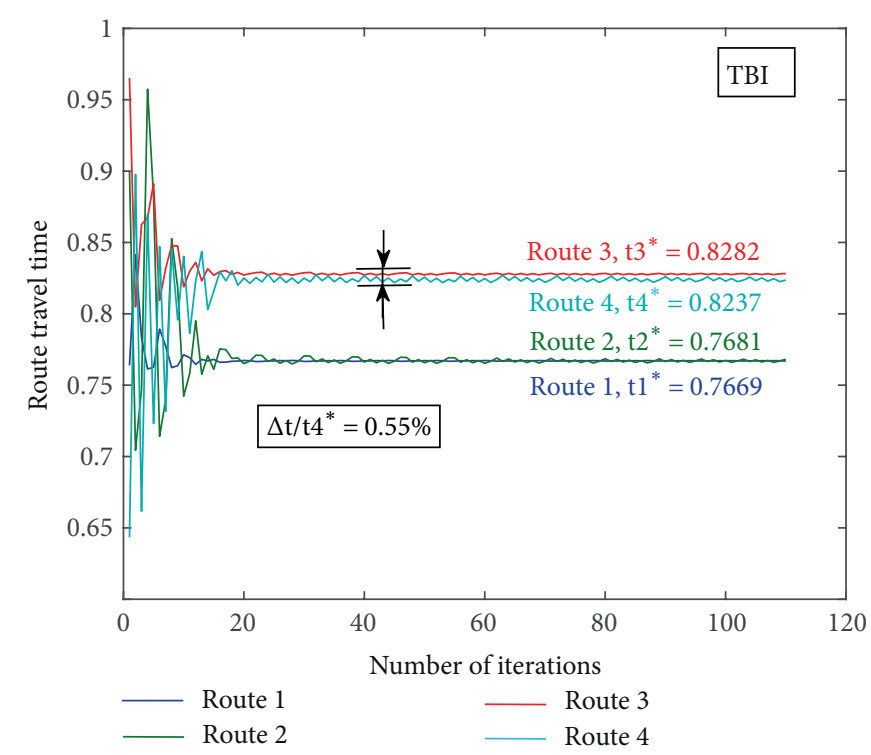

(b)

FIgure 6: Convergence curves of ARTT of TPI and TBI $(\mathrm{Z} 1, s=1)$. (a) Convergence curves of ARTT of TPI (Z1, $s=1)$. (b) Convergence curves of PRTT of TBI $(\mathrm{Z} 1, s=1)$.

pair 1-4) that increasing travelers incline to prefer "weatherresistant" route R1 to R2 (671-1050-1353-1500, the total traffic demand is 1500). Such results are expected that with the worsening of the adverse weather scenarios, the travel time of "weather-sensitive" routes increase more sharply than that of "weather-resistant" ones, and travelers have a preference to those "weather-resistant" routes rather than "weather-sensitive" ones to evade higher travel costs. Note that this conclusion is drawn on the premise that it is widely believed by TBI that the weather forecast is relatively accurate (shown as cases Z1-Z4 in Table 6).

\subsubsection{Impact of Accuracy of Weather Forecast on Route Choice} (Z5/Z6). Recall that in Table 6 cases $Z 5$ and Z6 are designed to investigate the impact of weather forecast accuracy on travelers' route choice behavior, and heavy rain $(s=3)$ was assumed to be the scenario with the highest probability of occurrence. The equilibrium flow patterns in Z5 \& Z6 are presented in Figures 7(e) and 7(f). There are not large differences in the total link flows (denoted as black numbers in the links) of the network between Z5 (when the weather forecast is accurate) and Z6 (when the weather forecast is less accurate). Actually, on one hand, whether the weather forecast is accurate or not will not directly affect the route choice of TPI, because TPI would always acquire the actual route travel time of the network with the aid of ATIS. On the other hand, the calculated posterior probabilities in the hypothetic cases Z5 (0.99) and Z6 (0.90) have approximate values, which implies that in some cases if the occurring probability of some scenario reported from weather forecast is relatively high, the posterior probabilities of TBI calculated via the Bayes formula would have little impact on their PRTT.

\subsection{Sioux Falls Network}

5.2.1. Network Descriptions. In this section, our model and algorithm are applied and tested to a well-known middle-size traffic network, i.e., the Sioux Falls network (Figure 8(a)), which has been frequently used as a numerical example in the field of transportation network modeling. The Sioux Falls network has 24 nodes, 76 links, and 552 O-D pairs. The general configurations of the network, including the free flow travel time and ideal link capacity, are consistent with reference [21,22], and further illustrated in Figure 8(b). The OD demand are the values from the Table 1 in [21] multiplied by 100. Given the complexity of the Sioux Falls network and to lower the computational cost, the link cost function in this case adopts the following reduced version, and the parameters are as previously stated:

$$
t_{a}^{s}\left(i_{s}, x_{a}\right)=g_{t}^{a}\left(i_{s}\right) t_{a}^{0}\left(1+\frac{x_{a}}{g_{c}^{a}\left(i_{s}\right) c_{a}^{0}}\right) \quad \forall a \in A, s \in S .
$$

5.2.2. Network Parameter Settings. In this section, to investigate the impact of links with different levels of adverse weather sensitivity on travelers' route choice behavior, the Sioux Falls network is divided into three parts, i.e., the weather-sensitive area $A_{s}$ (denoted in red dashed lines), weather-neutral area $A_{n}$ (in blue dashed lines) and weather-resistant area $A_{r}$ (in green dashed lines) as shown in Figure 8(b). The absolute value of link parameters $\eta$ and $\tau$ in the weather-sensitive area $(0.07$ and 0.04 , respectively) are larger than those of the other two areas, which indicates that for a given increment of rainfall intensity $\Delta i_{s}$ and all things being equal, the link travel time of links in weather-sensitive areas increase more sharply than those of links in other two areas.

Two rainfall scenarios, i.e., moderate rain $\left(s^{\prime}=1\right)$ and rainstorm $\left(s^{\prime}=2\right)$, are assumed in this case, and the corresponding rainfall intensities and the perceived conditional probability distributions of the TBI are shown in Tables 9 and 10, respectively. In this case, the TBI are assumed to hold positive attitudes towards the accuracy of the weather forecast. 


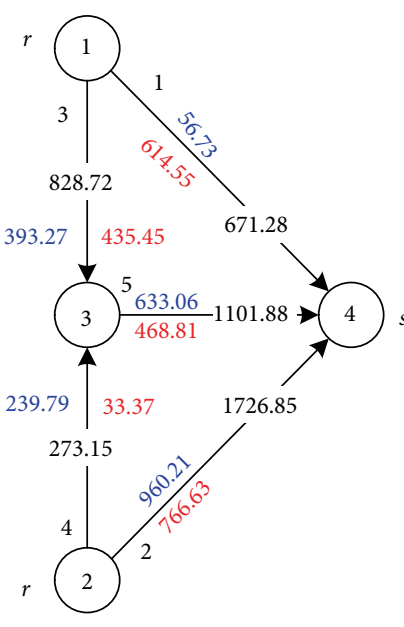

$\mathrm{Z1}\left(s=1\right.$, small rain, $\left.i_{1}=2.5\right)$

(a)

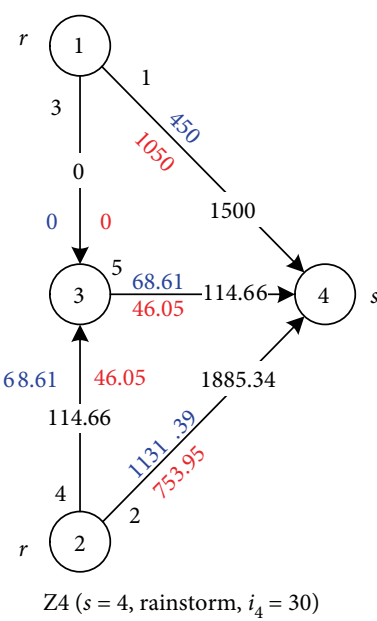

(d)

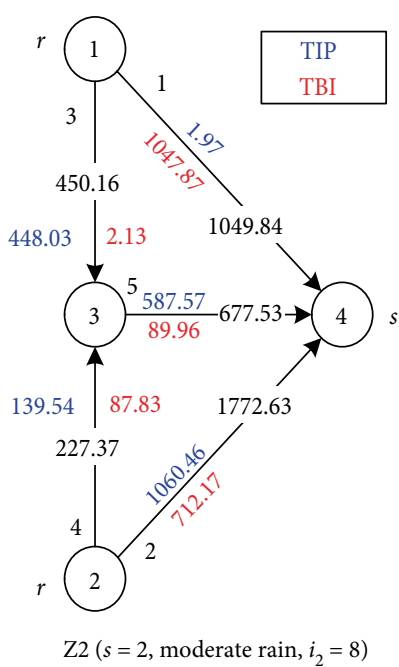

(b)

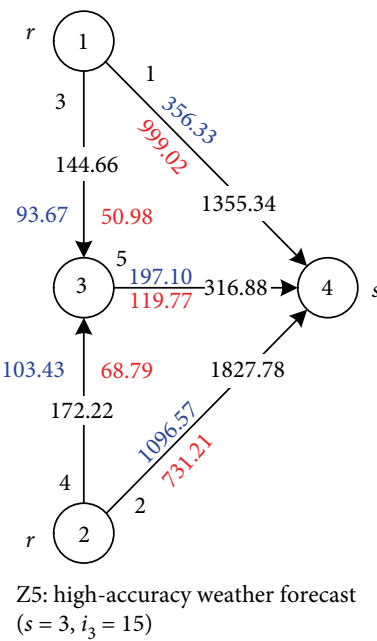

(e)

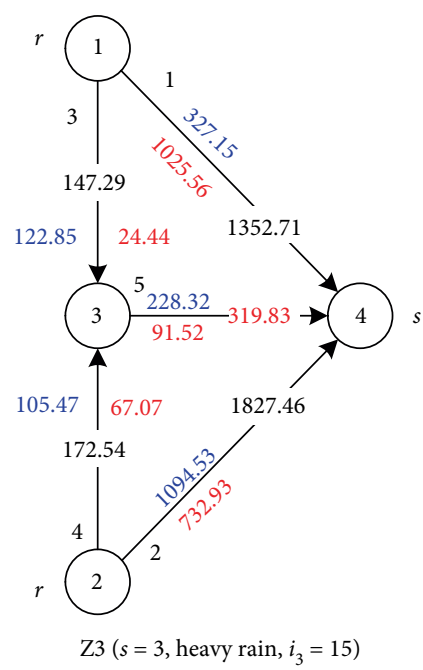

(c)

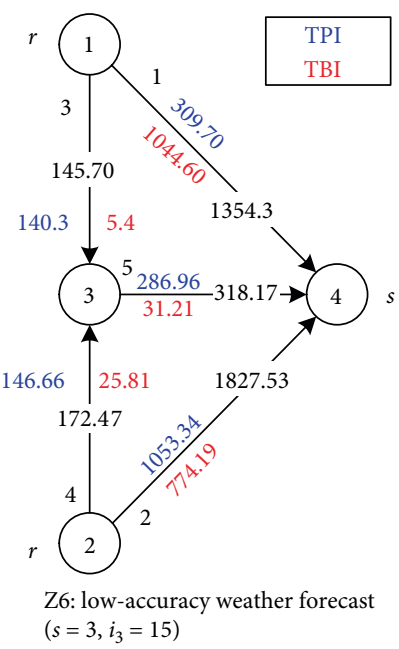

(f)

Figure 7: Equilibrium flow patterns in Z1-Z6.

5.2.3. Results and Sensitivity Analysis. For scenario Z7 (TPI: TBI $=60 \%: 40 \%)$, the algorithm converged after $n=47$ iterations. The iteration step size $\lambda_{n}$ and the convergence gap $e$ are shown in Figure 9. The missing points of the convergence gap curve at the beginning of the iterations indicate a positive infinite value of the convergence gap $e$.

The equilibrium flow pattern of five directed links for each of the three areas (i.e., $A_{s}, A_{n}$, and $A_{r}$ ) in case Z7 and Z8 (TPI:TBI $=60 \%: 40 \%$ ) in the network are reported in Table 11. The five links in one area are selected as spatially multidirectional as possible. To capture the variation tendency of the flows when the adverse weather scenario worsens from moderate rain to rainstorm, two rightward links (i.e., $(3,4)$, $(13,24))$ are selected in the leftmost area $A_{s}$ in Figure $8(\mathrm{~b})$, and for the rightmost area $A_{r}$, two leftward links (i.e., $(7,8)$, $(20,22))$ are selected.

We can learn from Table 11 that, when the weather scenario worsens, i.e., varies from moderate rain (case Z7) to rainstorm (case Z8), the flows of both TPI and TBI on the five selected links in the weather-sensitive area $A_{s}$ decrease by $15-46 \%$. While the flows of the two-class users in the weather-resistant area $A_{r}$ increase by $9-42 \%$. The OD demand transferred from the weather-sensitive area $A_{s}$ superimpose those local demand of the weather-neutral area $A_{n}$, leading to the flow of TPI and TBI on the selected rightward links $(1,2)$ connecting the $A_{s}$ and $A_{r}$ increase by $46.1 \%$ and $42 \%$, respectively. In the case of $Z 8$ (i.e., rainstorm), for the increasing travel cost in the areas $A_{s}$ nd $A_{n}$, the link travel costs in the weather-resistant area $A_{r}$ increase relatively more slowly and more preferred by travelers. Other results we can obtain are, for the weather-sensitive area $A_{s}$ and weather-resistant area $A_{r}$, the proportions of flow increment or decrement of TPI are larger than those of TBI. With the help of ATIS, TPI are more sensitive to the increasing actual link travel time in the weather-sensitive area $A_{s}$ and react faster than TBI. However, as a middle traversing area, the mixing effect makes it not always the case. For example, the proportions of four flow-decreasing links (i.e., $(15,22),(10$, $9),(10,11)$ and $(23,22))$ of TPI are smaller than those of TBI. 


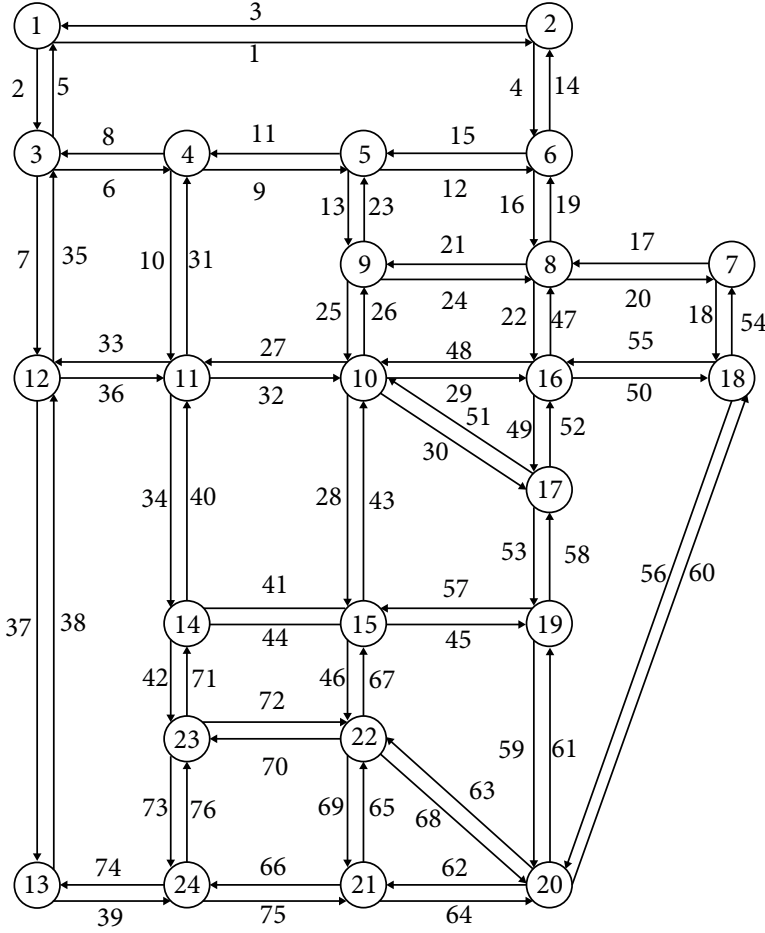

(a)

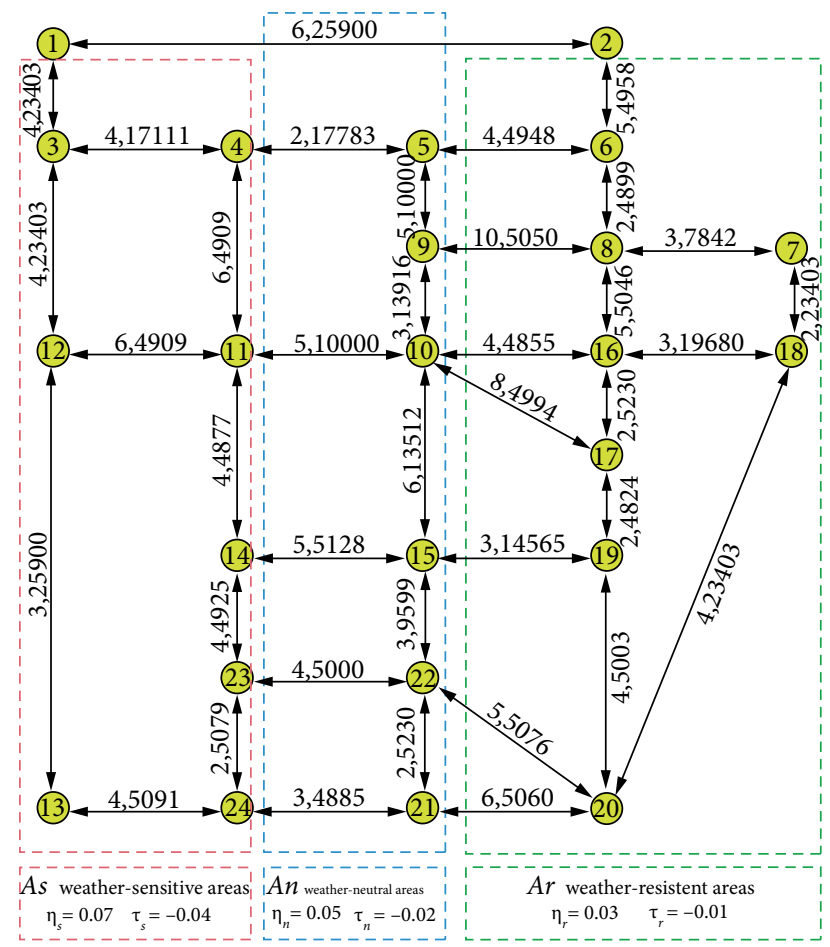

(b)

FIgURE 8: Sioux Falls network. (a) Numbers of nodes and links. (b) Link free flow travel time and ideal link capacity.

TABLE 9: Network scenarios and corresponding intensities.

\begin{tabular}{lc}
\hline Scenarios $s^{\prime}$ & Rainfall intensities $i_{s},(\mathrm{~mm} / \mathrm{h})$ \\
\hline Moderate rain $\left(s^{\prime}=1\right)$ & 8 \\
Rainstorm $\left(s^{\prime}=2\right)$ & 30 \\
\hline
\end{tabular}

TABLE 10: Different occurring probability (OP) and conditional probability distribution of TBI.

\begin{tabular}{|c|c|c|c|c|c|c|}
\hline \multirow{3}{*}{ Case } & \multirow{2}{*}{\multicolumn{2}{|c|}{$\begin{array}{l}\text { OP from weather } \\
\text { forecast: } p_{s}(\%)\end{array}$}} & \multicolumn{4}{|c|}{$\begin{array}{l}\text { Conditional probability of } \\
\qquad p_{s}: d_{p_{s} / s}(\%)\end{array}$} \\
\hline & & & \multicolumn{2}{|c|}{ TPI } & \multicolumn{2}{|c|}{ s $\quad$ TBI } \\
\hline & $\breve{p}_{1}$ & $\breve{p}_{2}$ & $d_{\vec{p}_{1} / 1}^{1}$ & $d_{\vec{p}_{2} / 2}^{1}$ & $d_{p_{1} / 1}^{2}$ & $d_{\bar{p}_{2} / 2}^{2}$ \\
\hline $\mathrm{Z7}$ & 90 & 10 & 100 & 0 & 90 & 10 \\
\hline Z8 & 30 & 70 & 0 & 100 & 30 & 70 \\
\hline
\end{tabular}

Besides the aforementioned results, we also care about how the proportion of the two classes of travelers impacts the network travel costs under the adverse weather scenarios. In other words, whether the increasing usage of ATIS, i.e, more percentage of TPI in the network, would increase drivers' perceived link travel cost. We adjust the share of the TPI in the two-class network, and depict the link travel time in Figure 10. As the proportion of TPI in the network increases from $20 \%, 40 \%, 60 \%$ to $80 \%$, the perceived link travel time of TPI rise with varying degrees. If the users in the network are more likely to be smarter and smarter with ATIS, more travelers

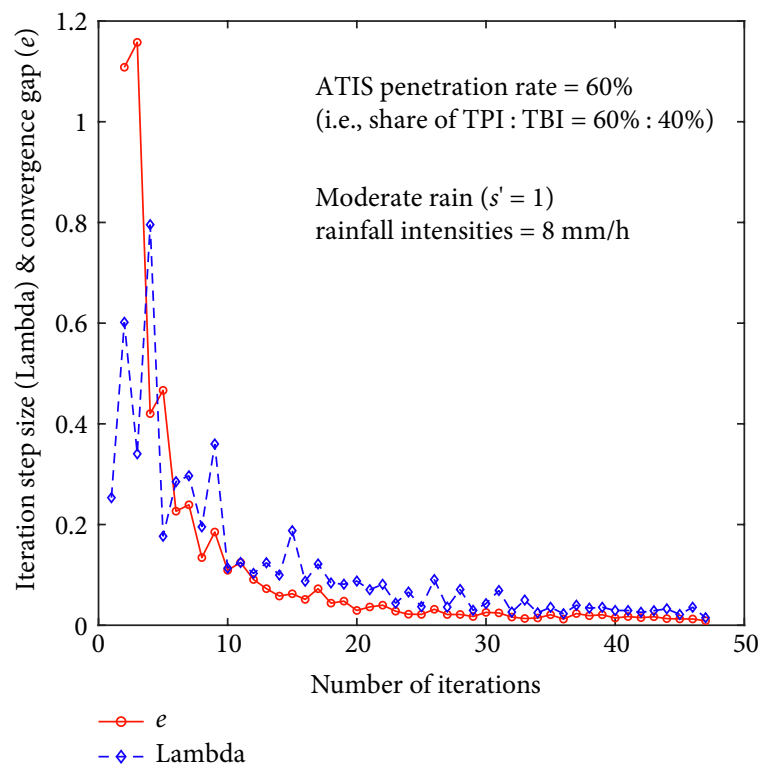

Figure 9: Convergence curves in case Z7.

can learn about the actual link travel cost in the network. Under the adverse weather conditions, when more smart users are guided to the recommended "shortest" routes, the perceived link travel cost also increases, and the advantages of ATIS in a low-proportion smart network could be weakened. 
TABLE 11: The equilibrium flow patterns of TPI/TBI in Sioux Falls network in case Z7 and Z8.

\begin{tabular}{|c|c|c|c|c|c|c|c|c|}
\hline \multirow{3}{*}{$\begin{array}{l}\text { [TPI: } \\
\text { Areas }\end{array}$} & \multirow{3}{*}{$\frac{\text { Directions }}{\downarrow}$} & \multirow{3}{*}{$\frac{\text { Links }}{(12,13)}$} & \multicolumn{2}{|c|}{ Z7 (moderate rain) } & \multicolumn{4}{|c|}{ Z8 (rainstorm) } \\
\hline & & & TPI & TBI & \multicolumn{2}{|c|}{$\operatorname{TPI}(\Delta \%)$} & \multicolumn{2}{|c|}{ TBI $(\Delta \%)$} \\
\hline & & & 7991 & 5155 & 6581 & $-17.6 \%$ & 4352 & $-15.6 \%$ \\
\hline & $\uparrow$ & $(14,11)$ & 4929 & 3112 & 2703 & $-45.2 \%$ & 1864 & $-40.1 \%$ \\
\hline \multirow[t]{5}{*}{$A_{s}$} & $\rightarrow$ & $(3,4)$ & 8095 & 5269 & 5927 & $-26.8 \%$ & 4001 & $-24.1 \%$ \\
\hline & $\leftarrow$ & $(11,12)$ & 4436 & 2851 & 2939 & $-33.7 \%$ & 1993 & $-30.1 \%$ \\
\hline & $\rightarrow$ & $(13,24)$ & 6233 & 4002 & 4768 & $-23.5 \%$ & 3223 & $-19.5 \%$ \\
\hline & $\downarrow$ & $(15,22)$ & 11220 & 7634 & 9831 & $-12.4 \%$ & 6578 & $-13.8 \%$ \\
\hline & $\uparrow$ & $(10,9)$ & 12719 & 8526 & 11746 & $-7.6 \%$ & 7829 & $-8.2 \%$ \\
\hline \multirow[t]{5}{*}{$A_{n}$} & $\rightarrow$ & $(1,2)$ & 5172 & 3455 & 7555 & $46.1 \%$ & 4904 & $42.0 \%$ \\
\hline & $\leftarrow$ & $(10,11)$ & 9710 & 6462 & 9169 & $-5.6 \%$ & 6091 & $-5.7 \%$ \\
\hline & $\rightarrow$ & $(23,22)$ & 5442 & 3520 & 5856 & $7.6 \%$ & 3914 & $11.2 \%$ \\
\hline & $\downarrow$ & $(6,8)$ & 10361 & 7028 & 14624 & $41.2 \%$ & 9645 & $37.2 \%$ \\
\hline & $\uparrow$ & $(20,19)$ & 5163 & 3450 & 6005 & $16.3 \%$ & 3992 & $15.7 \%$ \\
\hline \multirow[t]{3}{*}{$A_{r}$} & $\leftarrow$ & $(7,8)$ & 8444 & 5762 & 11493 & $36.1 \%$ & 7540 & $30.9 \%$ \\
\hline & $\rightarrow$ & $(10,17)$ & 4606 & 3067 & 5069 & $10.1 \%$ & 3365 & $9.7 \%$ \\
\hline & $\leftarrow$ & $(20,22)$ & 4792 & 3239 & 6276 & $31.0 \%$ & 4156 & $28.3 \%$ \\
\hline
\end{tabular}

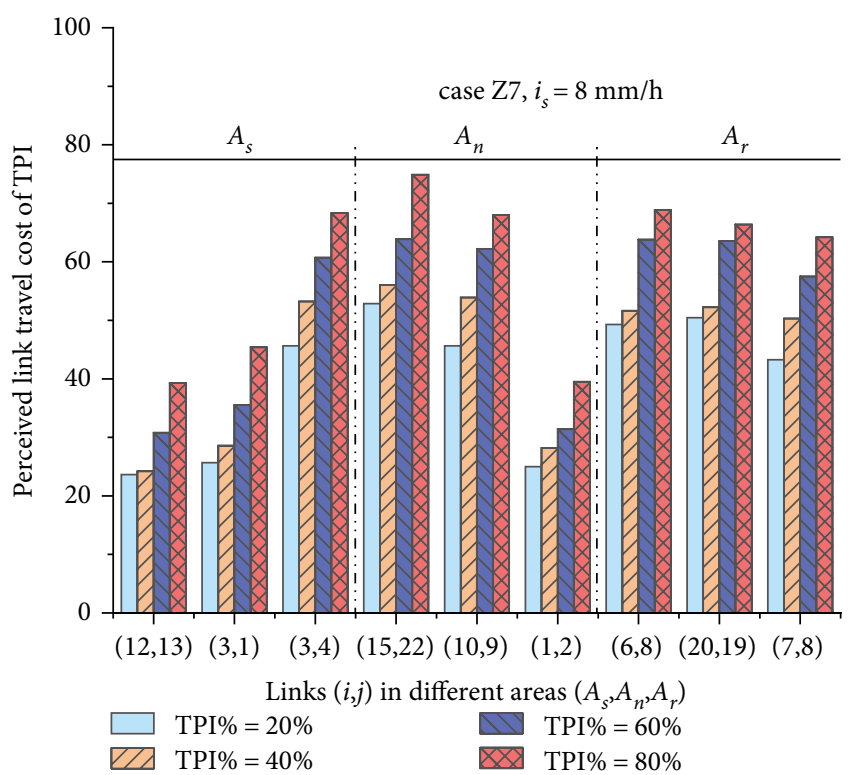

FIgURE 10: Perceived link travel time of TPI with different demand share in the network.

\section{Conclusions}

This paper focuses on the problem that how the adverse weather affects stochastic traffic network user equilibrium. We proposed a UE model incorporating the impacts of adverse weather scenarios on roadway performance degradation, and the influence of ATIS and weather forecast information on the route choice behavior of travelers with perfect information (TPI) and travelers with bounded information (TBI). Our consideration of the two-class user system is based on the discrepancy of network information level (NIL) of travelers, which divides the whole travelers into TPI who can obtain the actual network information with the help of ATIS and TBI who can just make their route choices based on their travel experience and perceptions on the accuracy of past weather forecast. The UE model was formulated as a VI problem and solved by a proposed algorithm. Numerical examples based on a simple triangle network and the well-known Sioux Falls network were adopted to verify the feature of the proposed model and solution algorithm, and to calculate the network equilibrium solutions.

From the results of the modeling and numerical analysis, several conclusions can be drawn as follows. The validity and feasibility of the proposed model and algorithm were testified. In the scenarios of more adverse weather intensities, more travelers (especially for TPI) are inclined to choose those "weather-resistant" routes to lower the travel time cost. From the point of the effect of ATIS on the system benefits, when the weather scenario is relatively good and the network capacity has not been deteriorated, ATIS can make travelers take full advantage of those high-capacity routes to shorten route travel time-the ATIS improve the overall system benefits. In contrast, when the weather scenario turns relatively adverse and the network capacity decreases rapidly, ATIS, however, guides travelers (especially for TPI and those TBI who consider the weather forecast information is accurate) to gather on those weather-resistant routes, in which the ATIS worsens the overall system benefits. In other words, when more smarter users can learn about the actual link travel cost in the network, the advantages of ATIS in a low-proportion smart network could be weakened.

Along with the deep research on stochastic network traffic assignment and equilibrium problem under adverse weather conditions, future research can be extended based on this study as follows. Besides TPI, given that different TBI with discrepant travel experience, homogeneous NILs in this study can be subdivided to heterogeneous levels, in which different TBI may have different perceptions on the accuracy of the same weather forecast information. What's more, the travel demand could be further considered as a decreasing function 
of the adverse weather intensity or route travel time, in which the deterministic travel demand problem is expanded to an elastic one. Finally, other expansions can lie in the consideration of travel time uncertainty, or developing more efficient VI solution algorithms.

\section{Appendix}

The proof below is given for Theorem 1 that the equilibrium conditions (18), (19) of the network are in accordance with the solutions of the VI problem (20).

Proof. The generalization of this proof referred to [9].

(1) Sufficiency. According to equilibrium conditions (18), (19) for TPI and TBI.

$$
\begin{aligned}
& \left(t_{1 r w}^{s}\left(\tilde{\boldsymbol{x}}^{*}\right)-\zeta_{1 w}^{s *}\right) \times\left(f_{1 r w}^{s}-f_{1 r w}^{s *}\right) \geq 0 \\
& \forall r \in R_{w}, w \in W, s \in S, \\
& \left(t_{2 r w}\left(\tilde{\boldsymbol{x}}^{*}\right)-\zeta_{2 w}^{s *}\right) \times\left(f_{2 r w}^{s}-f_{2 r w}^{s *}\right) \geq 0 \\
& \forall r \in R_{w}, \forall w \in W, \forall s \in S .
\end{aligned}
$$

Add the two inequations together, then sum for all routes $r \in R_{w}$, By using $d_{1 w}^{s}=\sum_{r \in R_{w}} f_{1 r w}^{s}=\sum_{r \in R_{w}} f_{1 r w}^{s *}$ and $d_{2 w}^{s}=\sum_{r \in R_{w}} f_{2 r w}^{s}=\sum_{r \in R_{w}} f_{2 r w}^{s *}$, we obtain

$$
\begin{aligned}
& \sum_{r \in R_{w}}\left[t_{1 r w}^{s}\left(\tilde{\boldsymbol{x}}^{*}\right) \times\left(f_{1 r w}^{s}-f_{1 r w}^{s *}\right)+t_{2 r w}\left(\tilde{\boldsymbol{x}}^{*}\right) \times\left(f_{2 r w}^{s}-f_{2 r w}^{s *}\right)\right] \\
& \quad \geq 0 \quad \forall w \in W, \forall s \in S .
\end{aligned}
$$

Sum the inequation for all OD pairs $w \in W$ and all network scenarios $s \in S$, and by using (13)-(15), we have

$$
\begin{aligned}
\sum_{s} \sum_{a}\left[t_{a 1}^{s}\left(\tilde{\boldsymbol{x}}^{*}\right)\right. & \times\left(x_{a 1}^{s}-x_{a 1}^{s *}\right)+t_{a 2}\left(\tilde{\boldsymbol{x}}^{*}\right) \\
& \left.\times\left(x_{a 2}^{s}-x_{a 2}^{s *}\right)\right] \geq 0 \quad \forall s \in S
\end{aligned}
$$

which is exactly the vector quantity pattern of the VI problem, i.e., (21). The sufficiency is proved.

(2) then we consider the necessity.

Assume that $\boldsymbol{x}_{1}=\left[x_{a 1}^{s}\right]_{|A||S|}, \boldsymbol{x}_{2}=\left[x_{a 2}^{s}\right]_{|A||S|}$ are the solutions of the VI problem (i.e., satisfy (21)), but they does not satisfy the equilibrium conditions (i.e., (18), (19)), for those TBI who travel between O-D pair $w$, there exists path $n$, so that,

$$
\begin{aligned}
& t_{1 n w}^{s}>\zeta_{1 w}^{s *} \\
& f_{1 n w}^{s *}>0 \quad \forall n \in R_{w}, \forall w \in W, \forall s \in S, \\
& t_{2 n w}^{s}>\zeta_{2 w}^{*} \\
& f_{2 n w}^{s *}>0 \quad \forall n \in R_{w}, \forall w \in W
\end{aligned}
$$

Thus, let us shift a small amount of path flow from path $n \in R_{w}$ to the path $\left(z \in R_{w}\right)$ with its cost equal to minimum route travel time $\left(\zeta_{1 w}^{s *}\right.$ and $\left.\zeta_{2 w}^{s *}\right)$ in equilibrium then

$$
\begin{aligned}
& \sum_{s} \sum_{w} \sum_{n} t_{1 n w}^{*} f_{1 n w}^{s}+\sum_{s} \sum_{w} \sum_{n} t_{2 n w}^{*} f_{2 n w}^{s *}<\sum_{s} \sum_{w} \sum_{z} t_{2 z w}^{*} f_{1 z w}^{s *} \\
& +\sum_{s} \sum_{w} \sum_{z} t_{2 z w}^{*} f_{2 z w}^{*} \Rightarrow \sum_{s} f_{1 n w}^{s} \sum_{w} \sum_{n} \sum_{a} \delta_{a n w} t_{a 1}^{s *} \\
& +\sum_{s} f_{2 n w}^{s} \sum_{w} \sum_{n} \sum_{a} \delta_{a n w} t_{a 2}^{*}=\sum_{s} \sum_{a} t_{a 1}^{s *} x_{a 1}^{s} \\
& +\sum_{s} \sum_{a} t_{a 2}^{*} x_{a 2}^{s}<\sum_{s} \sum_{a} t_{a 1}^{s *} x_{a 1}^{s *}+\sum_{s} \sum_{a} t_{a 2}^{s} x_{a 2}^{s *} \\
& \text { i.e., } \quad \\
& \quad \sum_{s} \sum_{a}\left(t_{a 1}^{s *}\left(x_{a 1}^{s}-x_{a 1}^{s *}\right)+t_{a 2}^{s *}\left(x_{a 2}^{s}-x_{a 2}^{s *}\right)\right)<0
\end{aligned}
$$

This contradicts with the assumption. So the solutions of the VI problem, i.e., $x_{1}, x_{2}$ coincide with the equilibrium conditions of the stochastic traffic network. So the sufficiency is proved.

\section{Data Availability}

The data of the small-size triangle network used in this study is meticulously designed by the authors, while the configurations of the well-known Sioux Falls network can be obtained via http://www.bgu.ac.il/ bargera/tntp/ and some classical references that have been declared in the paper.

\section{Conflicts of Interest}

The authors declare that they have no conflicts of interest.

\section{Acknowledgments}

The authors are indebted to two anonymous reviewers for their instructive and valuable comments to this paper. This study is supported by the Fund of Cultivation Project for Young University Teachers from Shanghai Municipal Education Commission (grant number: ZZHS18001), the National Natural Science Foundation of China (grant number: 71602114), and Shanghai Science and Technology Committee (grant numbers 17040501700 and 19QA1404200).

\section{References}

[1] B. W. Siu and H. K. Lo, "Doubly uncertain transportation network: degradable capacity and stochastic demand," European Journal of Operational Research, vol. 191, no. 1, pp. 166-181, 2008.

[2] A. Chen and Z. Zhou, "The a-reliable mean-excess traffic equilibrium model with stochastic travel times," Transportation Research Part B: Methodological, vol. 44, no. 4, pp. 493-513, 2010.

[3] J. He and C. Tan, "Modelling a resilient yard template under storage demand fluctuations in a container terminal," Engineering Optimization, vol. 51, no. 9, pp. 1547-1566, 2019.

[4] W. Mi, W. Yan, J. He, and D. Chang, "An investigation into yard allocation for outbound containers," COMPEL-The International 
Journal for Computation and Mathematics in Electrical and Electronic Engineering, vol. 28, no. 6, pp. 1442-1457, 2009.

[5] H. Shao, W. H. Lam, and M. L. Tam, "A reliability-based stochastic traffic assignment model for network with multiple user classes under uncertainty in demand," Networks and Spatial Economics, vol. 6, no. 3-4, pp. 173-204, 2006.

[6] C. Jiang, J. John Lu, Y. Jiang, X. Cai, and A. Ni, "Developing a traffic management framework for coastal expressway bridges under adverse weather conditions: case study of rain day in Shenzhen, China," Discrete Dynamics in Nature and Society, vol. 2015, Article ID 218672, p. 11, 2015.

[7] S. D. Clark and D. P. Watling, "Modelling network travel time reliability under stochastic demand," Transportation Research Part B: Methodological, vol. 39, no. 2, pp. 119-140, 2005.

[8] K. Uchida, A. Sumalee, and H. W. Ho, "A stochastic multimodal reliable network design problem under adverse weather conditions," Journal of Advanced Transportation, vol. 49, no. 1, pp. 73-95, 2015.

[9] C. Xie and Z. Liu, "On the stochastic network equilibrium with heterogeneous choice inertia," Transportation Research Part B: Methodological, vol. 66, pp. 90-109, 2014.

[10] M. Ben-Akiva, M. Bierlaire, H. N. Koutsopoulos, and R. Mishalani, "Real time simulation of traffic demand-supply interactions within DynaMIT," in Transportation and Network Analysis: Current Trends, pp. 19-36, Springer, USA, 2002.

[11] A. Vitetta, G. Musolino, and F. A. Marcianò, "Safety of users in road evacuation: supply and demand-supply interaction models for users," WIT Transactions on the Built Environment, p. 96, 2007.

[12] R. B. Chen and H. S. Mahmassani, "Let it rain: weather effects on activity stress and scheduling behavior," Travel Behaviour and Society, vol. 2, no. 1, pp. 55-64, 2015.

[13] W. H. K. Lam, H. Shao, and A. Sumalee, "Modeling impacts of adverse weather conditions on a road network with uncertainties in demand and supply," Transportation Research Part B: Methodological, vol. 42, no. 10, pp. 890-910, 2008.

[14] H. Shao, W. H. Lam, M. L. Tam, and X. M. Yuan, "Modelling rain effects on risk-taking behaviors of multi-user classes in road networks with uncertainty," Journal of Advanced Transportation, vol. 42, no. 3, pp. 265-290, 2008.

[15] A. Sumalee, K. Uchida, and W. H. K. Lam, "Stochastic multimodal transport network under demand uncertainties and adverse weather condition," Transportation Research Part C: Emerging Technologies, vol. 19, no. 2, pp. 338-350, 2011.

[16] J. Wardrop, "Some theoretical aspects of road traffic research," Proceedings of the Institute of Civil Engineering, Part II, vol. 1, no. 2, pp. 325-378, 1952.

[17] A. Nagurney, "Springer science \& business media," Network Economics: A Variational Inequality Approach-Revised Second Edition, Springer, 1999.

[18] Y. Sheffi, Urban Transportation Networks: Equilibrium Analysis With Mathematical Programming Methods, Prentice Hall, 1985.

[19] M. Frank and P. Wolfe, "An algorithm for quadratic programming," Naval Research Logistics, vol. 3, no. 1-2, pp. 95-110, 1956.

[20] S. Dafermos, "Relaxation algorithms for the general asymmetric traffic equilibrium problem," Transportation Science, vol. 16, no. 2, pp. 231-240, 1982.

[21] L. J. LeBlanc, E. K. Morlok, and W. P. Pierskalla, "An efficient approach to solving the road network equilibrium traffic assignment problem," Transportation Research, vol. 9, no. 5, pp. 309-318, 1975.

[22] Z. Di, L. Yang, J. Qi, and Z. Gao, “Transportation network design for maximizing flow-based accessibility," Transportation Research Part B: Methodological, vol. 110, pp. 209-238, 2018. 